\title{
EXISTENCE AND UNIQUENESS OF PERIODIC SOLUTIONS FOR PARABOLIC EQUATION WITH NONLOCAL DELAY*
}

\author{
Qiang Li ${ }^{\dagger}$, Yongxiang Li AND Pengyu Chen
}

\begin{abstract}
This paper deals with the existence and uniqueness of time periodic solutions for the general periodic parabolic equation boundary problem with nonlocal delay. We apply operator semigroup theory and monotone iterative technique of lower and upper solutions to obtain the existence and uniqueness of $\omega$-periodic mild solutions of some abstract evolution equation under some quasimonotone conditions. In the end, applying our abstract results to parabolic equation with nonlocal delay, we get the existence and uniqueness of $\omega$-periodic solution, which generalize the recent conclusions on this issue.
\end{abstract}

\section{Introduction}

Parabolic partial equations have been intensively developed during the last decades because of their applications in chemistry, biology, etcetera. Especially, periodic semi-linear parabolic partial equations are of particular interests since they can take into account seasonal fluctuations occurring in the phenomena appearing in the models, and have been extensively studied by many researchers (see $[2,1,6,14,22]$ and so on).

Since equations with delay are often more realistic to describe natural phenomena than those without delay, the problems concerning periodic solutions of partial differential equations with delays are an important area of investigation in recent years. Specially, the existence of periodic solutions of parabolic equation with delays has attracted much attention and been considered by some authors, see $[26,8,5,16,19,20,28,23,27,13]$ and references therein.

2010 Mathematics Subject Classification. 34C25; 34K30; 47H07; 47H08.

Key words and phrases. Parabolic equation with nonlocal delay; Time periodic mild solutions; Existence and uniqueness; Semigroup of Operator; Lower and upper solutions; Monotone iterative technique.

* Research supported by NNSFs of China $(11261053,11361055,11501455)$ and Project of NWNU-LKQN-14-3.

$\dagger$ Corresponding author.

Received April 9, 2015; revised September 17, 2015. 
Let $\Omega \subset \mathbf{R}^{N}$ be a bounded domain with a sufficiently smooth boundary $\partial \Omega$, and

$$
A(x, D) u=-\sum_{i, j=1}^{N} a_{i j}(x) D_{i} D_{j} u+\sum_{j=1}^{N} a_{j}(x) D_{j} u+a_{0}(x) u
$$

be a uniformly elliptic differential operator in $\bar{\Omega}$, whose coefficients $a_{i j}(x), a_{j}(x)$ $(i, j=1, \ldots, N)$ and $a_{0}(x)$ are Hölder-continuous on $\bar{\Omega}$ and $a_{0}(x) \geq 0$. We let $B=B(x, D)$ be a boundary operator on $\partial \Omega$ of the form

$$
B u:=b_{0}(x) u+\delta \frac{\partial u}{\partial \beta},
$$

where either $\delta=0$ and $b_{0}(x) \equiv 1$ (Dirichlet boundary operator), or $\delta=1$ and $b_{0}(x) \geq 0$ (regular oblique derivative boundary operator, at this point, we further assume that $a_{0}(x) \not \equiv 0$ or $\left.b_{0}(x) \not \equiv 0\right), \beta$ is an outward pointing, nowhere tangent vector field on $\partial \Omega$. Let $f: \Omega \times \mathbf{R} \times \mathbf{R}^{2} \rightarrow \mathbf{R}$ be local Hölder-continuous function which is $\omega$-periodic in $t$.

The time $\omega$-periodic solution for delay parabolic boundary value problem

$$
\left\{\begin{array}{l}
\left.\frac{\partial u}{\partial t}+A(x, D) u=f(x, t, u(x, t), u(x, t-\tau))\right), \quad x \in \Omega, t \in \mathbf{R}, \\
B u=0, \quad x \in \partial \Omega
\end{array}\right.
$$

has been studied by some authors, where $\tau$ is positive constant which denotes the time delay. If $\tau=0$, it means that system (1.3) does not include the terms of time lag. Theorems of the existence and uniqueness of periodic solution for some special kinds of equation (1.3) are proved in [8, 20, 28, 23].

In fact, in many practical problems individuals usually move around. In this case, it is not sufficient only to include a discrete delay or some finite delays, since the individuals may be at the different locations in their history, for example, in some population models. Therefore, in order to describe these models better, Britton [3, 4] considered these two factors and introduced the so called spatio-temporal delay or nonlocal delay. Afterwards, lots of works have been done on reaction-diffusion equations with nonlocal delay, we refer to $[24,17,18,15]$. It is noteworthy that these research works focused on the traveling wave solutions of parabolic equation with nonlocal delay. However, to our knowledge, there are still very few papers to consider the periodic problem of parabolic equation with nonlocal delay.

It is well known that the method of lower and upper solutions is an effective and flexible mechanism. It yields monotone sequences of lower and upper approximate solutions that converge to the minimal and maximal solutions between the lower and upper solutions. Recently, the method of lower and upper solutions and the monotone iterative technique had been developed for abstract evolution equations with classical initial condition and periodic condition $[10,11,25,12]$. 
Motivated by the papers mentioned above, in this paper, by means of operator semigroup theory and monotone iterative technique of lower and upper solutions, we discuss the existence and uniqueness of time $\omega$-periodic solutions of nonlocal delayed parabolic equation boundary value problem

$$
\left\{\begin{array}{l}
\frac{\partial u}{\partial t}+A(x, D) u=f(x, t, u(x, t), g * u(x, t)), \quad x \in \Omega, t \in \mathbf{R}, \\
B u=0, \quad x \in \Omega
\end{array}\right.
$$

where $f: \Omega \times \mathbf{R} \times \mathbf{R}^{2} \rightarrow \mathbf{R}$ is local Hölder-continuous function, $f(x, t, \xi, \eta)$ is once growth for $\xi$ and $\eta$, and $\omega$-periodic in $t$, the convolution $g * u(x, t)$ is denoted by

$$
\begin{aligned}
g * u(x, t) & =\int_{-\infty}^{t} \int_{\Omega} K(x, y, t-s) u(x, s) d y d s \\
& =\int_{0}^{+\infty} \int_{\Omega} K(x, y, s) u(x, t-s) d y d s .
\end{aligned}
$$

The convolution kernel $K(x, y, t)$ depends on both the spatial and the temporal variables, which is a positive continuous function in its variables $t \in \mathbf{R}, x, y \in \Omega$. We normalize the kernel so that

$$
\int_{0}^{+\infty} \int_{\Omega} K(x, y, t) d y d s=1
$$

For example [7], we take $\Omega=[0, \pi] \subset \mathbf{R}, K(x, y, t)=G(x, y, t) k(t), k(t)=\frac{1}{\tau} e^{-t / \tau}$ and

$$
G(x, y, t)=\frac{1}{\pi}+\frac{2}{\pi} \sum_{n=1}^{\infty} e^{-n^{2} t} \cos n x \sin n y,
$$

which is a fundamental solution of the heat equation

$$
\left\{\begin{array}{l}
\frac{\partial G}{\partial t}=\frac{\partial^{2} G}{\partial y^{2}}, \quad y \in[0, \pi], t>0, \\
\left.\frac{\partial G}{\partial y}\right|_{y=0, \pi}=0 \\
G(x, y, 0)=\delta(x-y),
\end{array}\right.
$$

where $\delta$ is the general Dirac function.

The paper is organized as follows. In Section 2, we collect some known notions and results on the operator semigroup and provide preliminary results to be used in theorems stated and proved in the paper. In Section 3, we present our abstract results and apply the operator semigroup theory and monotone iterative method of the lower and upper solutions to prove them. In the last section, applying our abstract results to parabolic equation with nonlocal delay, we get the existence and uniqueness of $\omega$-periodic solution. 


\section{Preliminaries}

In this section, we introduce some notions and definitions which are needed to prove our main results.

Let $n<p<\infty$ and denote $E=L^{p}(\Omega)$. Then $E$ is a Banach space with the $L^{p}$-norm $\|\cdot\|_{p}$. Let the positive cone $K=\{u \in E \mid u(x) \geq 0, x \in \Omega\}$, it is clear that $K$ is a normal regeneration cone, which induces a partial ordering " $\geq$ " on $E$ defined by

$$
u \geq v \Leftrightarrow u-v \in K .
$$

Thus, $E$ is an ordered Banach space with the partial order " $\geq$ ".

Let $C_{\omega}(\mathbf{R}, E)$ denote the Banach space $\{u \in C(\mathbf{R}, E) \mid u(t+\omega)=u(t), t \in R\}$ endowed the maximum norm $\|u\|_{C}=\max _{t \in[0, \omega]}\|u(t)\|_{p}$. Evidently, $C_{\omega}(\mathbf{R}, E)$ is also an order Banach space with the partial order " $\leq$ " induced by the positive cone $K_{C}=\left\{u \in C_{\omega}(\mathbf{R}, E) \mid u(t) \geq \theta, t \in \mathbf{R}\right\}$ and $K_{C}$ is also normal. For any $\alpha, \beta \in$ $C_{\omega}(\mathbf{R}, E)$ with $\alpha \leq \beta$, we use $[\alpha, \beta]$ to denote the order interval $\{u \mid \alpha \leq u \leq \beta\}$ in $C_{\omega}(\mathbf{R}, E)$ and $[\alpha(t), \beta(t)]_{E}$ to denote the order interval $\{u(t) \mid \alpha(t) \leq u(t) \leq \beta(t)$, $t \in \mathbf{R}\}$ in $E$.

Define a linear operator $A: D(A) \rightarrow E$ by

$$
D(A)=\left\{u \in W^{2, p}(\Omega) \mid B(x, D) u=0, x \in \partial \Omega\right\}, \quad A u=A(x, D) u .
$$

If $a_{0}(x) \geq 0$, then $-A$ generates an exponentially stable analytic semigroup $T(t)(t \geq 0)$ in $E$ (see [2]). Let $\lambda_{1}$ be the first eigenvalue of $A(x, D)$ under the boundary condition $B u=0$, we know that $\lambda_{1}>0$. Thus, there is a constant $M_{0} \geq 1$ such that

$$
\|T(t)\| \leq M_{0} e^{-\lambda t} \leq M_{0}, \quad \text { for any } \lambda \in\left(0, \lambda_{1}\right), t \geq 0 .
$$

By the maximum principle of elliptic operators, we know that $\lambda I+A$ has positive bounded inverse operator $(\lambda I+A)^{-1}$ for $\lambda>0$, hence $T(t)(t \geq 0)$ is a positive semigroup (see [10]). Sine the operator $A$ has compact resolvent in $L^{p}(\Omega)$, we obtain that $T(t)(t \geq 0)$ is also a compact semigroup (see [9]). For more details of the properties of the operator semigroup, we refer to $[9,21]$.

Now, from the assumptions of $f$, we can define $F: \mathbf{R} \times E \times E \rightarrow E$ by

$$
F(t, u, v)=f(\cdot, t, u(\cdot), v(\cdot)),
$$

and $F: \mathbf{R} \times E \times E \rightarrow E$ is continuous and $\omega$-periodic for $t$. For any $u \in C_{\omega}(\mathbf{R}, E)$, $u(t) \in E=L^{p}(\Omega)$, denote $u(t)(x)=u(x, t)$, we can easily prove $g * u(x, t)=$ $g * u(x, t+\omega)$ for $x \in \Omega$, hence we can define an operator $S: C_{\omega}(\mathbf{R}, E) \rightarrow$ $C_{\omega}(\mathbf{R}, E)$ as follow

$$
S u=\int_{0}^{\infty} \int_{\Omega} K(x, y, s) u(x, t-s) d y d s .
$$

It is clear that $S: C_{\omega}(\mathbf{R}, E) \rightarrow C_{\omega}(\mathbf{R}, E)$ is a positive linear bounded operator with $\|S\| \leq 1$. Hence, the $\omega$-periodic problem of equation (1.4) can be reformulated 
as the $\omega$-periodic problem of abstract evolution equation

$$
u^{\prime}(t)+A u(t)=F(t, u(t), S u(t)), \quad t \in \mathbf{R} .
$$

Now, we recall some basic facts on abstract linear evolution equation corresponding to Eq. (2.5). Let $J$ denote the infinite interval $[0,+\infty)$ and $h: J \rightarrow E$, consider the initial value problem of the linear evolution equation

$$
\left\{\begin{array}{l}
u^{\prime}(t)+A u(t)=h(t), \quad t>0 \\
u(0)=x_{0}
\end{array}\right.
$$

It is well known [21], when $x_{0} \in E_{1}$ and $h \in C^{1}(J, E)$, the initial value problem (2.6) has a unique classical solution $u \in C^{1}(J, E) \cap C\left(J, E_{1}\right)$ expressed by

$$
u(t)=T(t) x_{0}+\int_{0}^{t} T(t-s) h(s) d s,
$$

where $E_{1}=D(A)$ is Banach space with the graph norm $\|\cdot\|_{1}=\|\cdot\|+\|A \cdot\|$. Generally, for $x_{0}$ and $h \in C(J, E)$, the function $u$ given by (2.6) belongs to $C(J, E)$ and it is called a mild solution of the linear evolution equation (2.6).

Given $h \in C_{\omega}(\mathbf{R}, E)$, we consider the existence of $\omega$-periodic mild solution of linear evolution equation

$$
u^{\prime}(t)+A u(t)=h(t), \quad t \in \mathbf{R} .
$$

In order to establish the results of this section, from [12] we include the following lemma.

Lemma 2.1. Assume that $A$ is defined by (2.1), thus $-A$ generates an exponentially stable analytic semigroup $T(t)(t \geq 0)$ in $E$. Then for $h \in C_{\omega}(\mathbf{R}, E)$, the linear evolution equation (2.8) has a unique w-periodic mild solution $u \in$ $C_{\omega}(\mathbf{R}, E)$, which can be expressed by

$$
u(t)=(I-T(\omega))^{-1} \int_{t-\omega}^{t} T(t-s) h(s) d s:=P h(t),
$$

and the solution operator $P: C_{\omega}(\mathbf{R}, E) \rightarrow C_{\omega}(\mathbf{R}, E)$ is a bounded positive linear operator with the spectral radius $r(P) \leq \frac{1}{\lambda_{1}}\left(\lambda_{1}\right.$ is the first eigenvalue of $\left.A\right)$.

Similarly to definition of $\omega$-periodic mild solution for the linear evolution equation (2.8), we can define the $\omega$-periodic mild solution for the nonlinear evolution equation (2.5) (or Eq. (1.4)).

Definition 2.1. A function $u \in C_{\omega}(\mathbf{R}, E)$ is said to be a mild solution of Eq. (2.5) if it satisfies

$$
u(t)=(I-T(\omega))^{-1} \int_{t-\omega}^{t} T(t-s) F(s, u(s), S u(s)) d s .
$$


Next, we define the $\omega$-periodic lower and upper solutions of Eq. (2.5).

Definition 2.2. If a function $\alpha \in C_{\omega}^{1}(\mathbf{R}, E) \cap C_{\omega}\left(\mathbf{R}, E_{1}\right)$ satisfies

$$
\alpha^{\prime}(t)+A \alpha(t) \leq F(t, \alpha(t), S \alpha(s)), \quad t \in \mathbf{R},
$$

we call it an $\omega$-periodic lower solution of Eq. (2.5). If the inequality in (2.11) is inverse, we call it an $\omega$-periodic upper solution of Eq. (2.5).

\section{Abstract results}

Now, we are in the position to state and prove our main results. We will apply monotone iterative method of the lower and upper $\omega$-periodic solutions to obtain the existence of $\omega$-periodic mild solution for Eq. (2.5).

THEOREM 3.1. Let $F: \mathbf{R} \times E \times E \rightarrow E$ is continuous function which is $\omega$-periodic in $t, S: C_{\omega}(\mathbf{R}, E) \rightarrow C_{\omega}(\mathbf{R}, E)$ is a positive linear bounded operator. Suppose that Eq. (2.5) has lower and upper w-periodic solutions $\alpha_{0}, \beta_{0} \in C_{\omega}^{1}(\mathbf{R}, E) \cap$ $C_{\omega}\left(\mathbf{R}, E_{1}\right)$ with $\alpha_{0} \leq \beta_{0}$. If the following assumptions

(A1) there are two constants $C_{1} \geq 0$ and $C_{2}>0$ such that

$$
F\left(t, w_{2}, v_{2}\right)-F\left(t, w_{1}, v_{1}\right) \geq C_{1}\left(w_{2}-w_{1}\right)+C_{2}\left(v_{2}-v_{1}\right),
$$

for all $t \in \mathbf{R}, \alpha_{0}(t) \leq w_{1} \leq w_{2} \leq \beta_{0}(t), S \alpha_{0}(t) \leq v_{1} \leq v_{2} \leq S \beta_{0}(t)$,

(A2) there is a constant $0 \leq C_{3} \leq \frac{C_{1}}{C_{2}}$ such that

$$
S w_{2}-S w_{1} \geq-C_{3}\left(w_{2}-w_{1}\right),
$$

for all $w_{1}, w_{2} \in\left[\alpha_{0}, \beta_{0}\right]$ and $w_{2} \geq w_{1}$,

hold, then Eq. (2.5) has minimal and maximal w-periodic mild solution $\underline{u}, \bar{u}$ between $\alpha_{0}$ and $\beta_{0}$, which can be obtained by monotone iterative sequences starting from $\alpha_{0}$ and $\beta_{0}$.

Proof. By the definition of lower and upper $\omega$-periodic solutions, we obtain that $\left[\alpha_{0}, \beta_{0}\right] \subset C_{\omega}(\mathbf{R}, E)$. It is easy to see that $\alpha_{0}(t) \leq \beta_{0}(t)$ for every $t \in \mathbf{R}$. Now, let us define $\mathscr{F}: C_{\omega}(\mathbf{R}, E) \rightarrow C_{\omega}(\mathbf{R}, E)$ by

$$
\mathscr{F}(u)(t)=F(t, u(t), S u(t)),
$$

where $F$ is defined by (2.4). We define the mapping $Q:\left[\alpha_{0}, \beta_{0}\right] \rightarrow C_{\omega}(\mathbf{R}, E)$ by

$$
Q u=(P \circ \mathscr{F})(u) \text {. }
$$

It is clear that

$$
(Q u)(t)=(I-T(\omega))^{-1} \int_{t-\omega}^{t} T(t-s) F(s, u(s), S u(s)) d s .
$$

We can easily see that $Q:\left[\alpha_{0}, \beta_{0}\right] \rightarrow C_{\omega}(\mathbf{R}, E)$ is continuous. By Definition 2.1 , the $\omega$-periodic mild solution of Eq. (2.5) is equivalent to the fixed point of operator $Q$. 
Firstly, we show that the following properties of $Q$.

(i) $Q$ is a monotone operator.

(ii) $\alpha_{0} \leq Q \alpha_{0}$ and $Q \beta_{0} \leq \beta_{0}$.

In fact, if $u_{1}, u_{2} \in\left[\alpha_{0}, \beta_{0}\right]$ and $u_{1} \leq u_{2}$, then $\alpha_{0}(t) \leq u_{1}(t) \leq u_{2}(t) \leq \beta_{0}(t)$ and $S \alpha_{0}(t) \leq S u_{1}(t) \leq S u_{2}(t) \leq S \beta_{0}(t)$ for every $t \in \mathbf{R}$, from $(A 1)$ and (A2), we obtain that

$$
\begin{aligned}
F(t, & \left.u_{2}(t), S u_{2}(t)\right)-F\left(t, u_{1}(t), S u_{1}(t)\right) \\
& \geq C_{1}\left(u_{2}(t)-u_{1}(t)\right)+C_{2}\left(S u_{2}(t)-S u_{1}(t)\right) \\
& \geq C_{1}\left(u_{2}(t)-u_{1}(t)\right)-C_{2} C_{3}\left(u_{2}(t)-u_{1}(t)\right) \\
& =\left(C_{1}-C_{2} C_{3}\right)\left(u_{2}(t)-u_{1}(t)\right) \geq 0 .
\end{aligned}
$$

Thus $F(t, u(t), G u(t))$ is nondecreasing on $u \in\left[\alpha_{0}, \beta_{0}\right]$. Namely,

$$
F\left(t, u_{1}(t), S u_{1}(t)\right) \leq F\left(t, u_{2}(t), S u_{2}(t)\right),
$$

for all $t \in \mathbf{R}$ and $u_{1}, u_{2} \in\left[\alpha_{0}, \beta_{0}\right]$ with $u_{1} \leq u_{2}$. Thus, by means of positivity of the operator semigroup $T(t)(t \geq 0)$, one has

$$
\begin{aligned}
(I- & T(\omega))^{-1} \int_{t-\omega}^{t} T(t-s) F\left(s, u_{1}(s), S u_{1}(s)\right) d s \\
& \leq(I-T(\omega))^{-1} \int_{t-\omega}^{t} T(t-s) F\left(s, u_{2}(s), S u_{2}(s)\right) d s .
\end{aligned}
$$

Hence, by (3.2), we see that $Q u_{1} \leq Q u_{2}$, which means that $Q$ is a monotone operator.

Let $h(t)=\alpha_{0}^{\prime}(t)+A \alpha_{0}(t) \in C_{\omega}(\mathbf{R}, E)$, by the definition of lower solution, we know that $h(t) \leq F\left(t, \alpha_{0}(t), S \alpha_{0}(t)\right)$ for $t \in \mathbf{R}$. Thus, form Lemma 2.1 and Definition 2.1, we have

$$
\begin{aligned}
\alpha_{0}(t) & =(I-T(\omega))^{-1} \int_{t-\omega}^{t} T(t-s) h(s) d s \\
& \leq(I-T(\omega))^{-1} \int_{t-\omega}^{t} T(t-s) F\left(s, \alpha_{0}(s), S \alpha_{0}(s)\right) d s \\
& =\left(Q \alpha_{0}\right)(t), \quad t \in \mathbf{R},
\end{aligned}
$$

namely, $\alpha_{0} \leq Q \alpha_{0}$. Similarly, it can be shown that $Q \beta_{0} \leq \beta_{0}$. Therefore, $Q:\left[\alpha_{0}, \beta_{0}\right] \rightarrow\left[\alpha_{0}, \beta_{0}\right]$ is a continuously increasing operator. scheme

Now, we define two sequences $\left\{\alpha_{n}\right\}$ and $\left\{\beta_{n}\right\}$ in $\left[\alpha_{0}, \beta_{0}\right]$ by the iterative

$$
\alpha_{n}=Q \alpha_{n-1}, \quad \beta_{n}=Q \beta_{n-1}, \quad n=1,2, \ldots
$$

Then from the monotonicity of the operator $Q$, it follows that

$$
\alpha_{0} \leq \alpha_{1} \leq \alpha_{2} \leq \cdots \leq \alpha_{n} \leq \cdots \leq \beta_{n} \leq \cdots \leq \beta_{2} \leq \beta_{1} \leq \beta_{0} .
$$


Secondly, we prove that $\left\{\alpha_{n}\right\}$ and $\left\{\beta_{n}\right\}$ are convergent in $C_{\omega}(\mathbf{R}, E)$.

Since $u \in\left[\alpha_{0}, \beta_{0}\right]$ implies that $\alpha_{0}(t) \leq u(t) \leq \beta_{0}(t)$ for any $t \in \mathbf{R}$, thus, from (3.3) we have

$$
F\left(t, \alpha_{0}(t), S \alpha_{0}(t)\right) \leq F(t, u(t), S u(t)) \leq F\left(t, \beta_{0}(t), S \beta_{0}(t)\right), \quad t \in \mathbf{R} .
$$

By the normality of the cones, there exist a constant $M_{1}>0$ such that

$$
\|F(t, u(t), S u(t))\| \leq M_{1}, \quad t \in \mathbf{R}, u \in\left[\alpha_{0}, \beta_{0}\right] .
$$

Now, we demonstrate that $\left\{\alpha_{n}\right\}$ and $\left\{\beta_{n}\right\}$ are equicontinuous.

For every $\alpha_{n}$, by the periodicity, we only consider it on $[0, \omega]$. Set $0 \leq t_{1}<$ $t_{2} \leq \omega$, we get that

$$
\begin{aligned}
\alpha_{n}\left(t_{2}\right)-\alpha_{n}\left(t_{1}\right)= & \left(Q \alpha_{n-1}\right)\left(t_{2}\right)-\left(Q \alpha_{n-1}\right)\left(t_{1}\right) \\
= & (I-T(\omega))^{-1} \int_{t_{2}-\omega}^{t_{2}} T\left(t_{2}-s\right) F\left(s, \alpha_{n-1}(s), S \alpha_{n-1}(s)\right) d s \\
& -(I-T(\omega))^{-1} \int_{t_{1}-\omega}^{t_{1}} T\left(t_{1}-s\right) F\left(s, \alpha_{n-1}(s), S \alpha_{n-1}(s)\right) d s \\
= & (I-T(\omega))^{-1} \int_{t_{2}-\omega}^{t_{1}}\left(T\left(t_{2}-s\right)-T\left(t_{1}-s\right)\right) F\left(s, \alpha_{n-1}(s), S \alpha_{n-1}(s)\right) d s \\
& -(I-T(\omega))^{-1} \int_{t_{1}-\omega}^{t_{2}-\omega} T\left(t_{1}-s\right) F\left(s, \alpha_{n-1}(s), S \alpha_{n-1}(s)\right) d s \\
& +(I-T(\omega))^{-1} \int_{t_{1}}^{t_{2}} T\left(t_{2}-s\right) F\left(s, \alpha_{n-1}(s), S \alpha_{n-1}(s)\right) d s \\
:= & I_{1}+I_{2}+I_{3} .
\end{aligned}
$$

It is clear that

$$
\left\|(Q u)\left(t_{2}\right)-(Q u)\left(t_{1}\right)\right\| \leq\left\|I_{1}\right\|+\left\|I_{2}\right\|+\left\|I_{3}\right\| .
$$

Hence, we only need to check $\left\|I_{i}\right\|$ tend to 0 independently of $\alpha_{n}$ when $t_{2}-t_{1} \rightarrow 0, i=1,2,3$. From [12], we know that $(I-T(\omega))^{-1}$ is a bounded operator, denote $\left\|(I-T(\omega))^{-1}\right\|=M_{2}$. Therefore, by (2.2) and (3.7), we can easily obtain

$$
\begin{aligned}
\left\|I_{1}\right\| & \leq M_{2} \int_{t_{2}-\omega}^{t_{1}}\left\|\left(T\left(t_{2}-s\right)-T\left(t_{1}-s\right)\right) F\left(s, \alpha_{n-1}(s), S \alpha_{n-1}(s)\right)\right\| d s \\
& \leq M_{1} M_{2} \int_{0}^{t_{1}+\omega-t_{2}}\left\|T\left(t_{2}-t_{1}+s\right)-T(s)\right\| d s \\
& \rightarrow 0, \quad \text { as } t_{2}-t_{1} \rightarrow 0,
\end{aligned}
$$




$$
\begin{aligned}
\left\|I_{2}\right\| & \leq M_{2} \int_{t_{1}-\omega}^{t_{2}-\omega}\left\|T\left(t_{1}-s\right) F\left(s, \alpha_{n-1}(s), S \alpha_{n-1}(s)\right)\right\| d s \\
& \leq M_{1} M_{2} \int_{t_{1}-\omega}^{t_{2}-\omega}\left\|T\left(t_{1}-s\right)\right\| d s \\
& \rightarrow 0, \quad \text { as } t_{2}-t_{1} \rightarrow 0 \\
\left\|I_{3}\right\| & \leq M_{2} \int_{t_{1}}^{t_{2}}\left\|T\left(t_{2}-s\right) F\left(s, \alpha_{n-1}(s), S \alpha_{n-1}(s)\right)\right\| d s \\
& \leq M_{1} M_{2} \int_{t_{1}}^{t_{2}}\left\|T\left(t_{2}-s\right)\right\| d s \\
& \rightarrow 0, \quad \text { as } t_{2}-t_{1} \rightarrow 0
\end{aligned}
$$

As a result, $\left\|\left(Q \alpha_{n-1}\right)\left(t_{2}\right)-\left(Q \alpha_{n-1}\right)\left(t_{1}\right)\right\|$ tends to 0 independently of $\alpha_{n}$ as $t_{2}-t_{1} \rightarrow 0$, which means that $\left\{\alpha_{n}\right\}$ is equicontinuous. Similarly, we can prove that $\left\{\beta_{n}\right\}$ is equicontinuous.

Next, we prove that $\left\{\alpha_{n}(t)\right\}$ and $\left\{\beta_{n}(t)\right\}$ are relatively compact in $E$ for all $t \in \mathbf{R}$. For convenience, let $B_{1}=\left\{\alpha_{n}\right\}, B_{2}=\left\{\beta_{n}\right\}$ and $B_{1}^{0}=B_{1} \cup\left\{\alpha_{0}\right\}, B_{2}^{0}=$ $B_{2} \cup\left\{\beta_{0}\right\}$, it follows that $B_{1}(t)=\left(Q B_{1}^{0}\right)(t)$ and $B_{2}(t)=\left(Q B_{2}^{0}\right)(t)$ for every $t \in \mathbf{R}$.

We define a set by

$$
\left(Q_{\varepsilon} B_{1}^{0}\right)(t):=\left\{\left(Q_{\varepsilon} \alpha_{n}\right)(t) \mid \alpha_{n} \in B_{n}^{0}, 0<\varepsilon<\omega, t \in \mathbf{R}\right\}
$$

where

$$
\begin{aligned}
Q_{\varepsilon} \alpha_{n}(t) & =(I-T(\omega))^{-1} \int_{t-\omega}^{t-\varepsilon} T(t-s) F\left(s, \alpha_{n-1}(s), S \alpha_{n-1}(s)\right) d s \\
& =T(\varepsilon)(I-T(\omega))^{-1} \int_{t-\omega}^{t-\varepsilon} T(t-s-\varepsilon) F\left(s, \alpha_{n-1}(s), S \alpha_{n-1}(s)\right) d s
\end{aligned}
$$

Then the set $\left(Q_{\varepsilon} B_{1}^{0}\right)(t)$ is relatively compact in $E$ since the operator $T(\varepsilon)$ is compact in $E$. For any $\alpha_{n} \in B_{1}^{0}$ and $t \in \mathbf{R}$, from the follow inequality

$$
\begin{aligned}
&\left\|Q \alpha_{n}(t)-Q_{\varepsilon} \alpha_{n}(t)\right\| \\
& \leq \|(I-T(\omega))^{-1} \int_{t-\omega}^{t} T(t-s) F\left(s, \alpha_{n-1}(s), S \alpha_{n-1}(s)\right) d s \\
& \quad-(I-T(\omega))^{-1} \int_{t-\omega}^{t-\varepsilon} T(t-s) F\left(s, \alpha_{n-1}(s), S \alpha_{n-1}(s)\right) d s \| \\
& \leq M_{2} \int_{t-\varepsilon}^{t}\left\|T(t-s) F\left(s, \alpha_{n-1}(s), S \alpha_{n-1}(s)\right)\right\| d s \\
& \leq M_{1} M_{2} \int_{t-\varepsilon}^{t}\|T(t-s)\| d s \leq M_{0} M_{1} M_{2} \varepsilon
\end{aligned}
$$


one can obtain that the set $\left(Q B_{1}^{0}\right)(t)$ is relatively compact in $E$ for all $t \in \mathbf{R}$. Thus $\left\{\alpha_{n}(t)\right\}=B_{1}(t)=\left(Q B_{1}^{0}\right)(t)$ is relatively compact in $E$ for all $t \in \mathbf{R}$. Similarly, we can prove that $\left\{\beta_{n}(t)\right\}$ is relatively compact in $E$ for all $t \in \mathbf{R}$.

Therefore, the Arzela-Ascoli theorem guarantees that $\left\{\alpha_{n}\right\}$ and $\left\{\beta_{n}\right\}$ are relatively compact in $C_{\omega}(\mathbf{R}, E)$, so there are convergent subsequences in $\left\{\alpha_{n}\right\}$ and $\left\{\beta_{n}\right\}$, respectively. Combining this with the monotonicity (3.5) and the normality of the cone $K$, we can easily prove that $\left\{\alpha_{n}\right\}$ and $\left\{\beta_{n}\right\}$ themselves are convergent, i.e., there are $\underline{u}, \bar{u} \in C_{\omega}(\mathbf{R}, E)$ such that $\lim _{n \rightarrow \infty} \alpha_{n}=\underline{u}$ and $\lim _{n \rightarrow \infty} \beta_{n}=\bar{u}$. Taking limit in (3.4), we have

$$
\underline{u}=Q \underline{u}, \quad \bar{u}=Q \bar{u} .
$$

Therefore $\underline{u}, \bar{u} \in C_{\omega}(\mathbf{R}, E)$ are fixed points of $Q$.

Finally, we prove the minimal and maximal properties of $\underline{u}, \bar{u}$. Assume that $\tilde{u}$ is a fixed point of $Q$ with $\tilde{u} \in\left[\alpha_{0}, \beta_{0}\right]$, then for every $t \in \mathbf{R}, \alpha_{0}(t) \leq \tilde{u}(t) \leq \beta_{0}(t)$,

$$
\alpha_{1}(t)=\left(Q \alpha_{0}\right)(t) \leq(Q \tilde{u})(t)=\tilde{u}(t) \leq\left(Q \beta_{0}\right)(t)=\beta_{1}(t), \quad t \in \mathbf{R} .
$$

Similarly, $\alpha_{1}(t) \leq \tilde{u}(t) \leq \beta_{1}(t)$ for $t \in \mathbf{R}$. In general

$$
\alpha_{n} \leq \tilde{u} \leq \beta_{n}, \quad n=1,2, \ldots
$$

Taking limit in (3.13) as $n \rightarrow \infty$, we get $\underline{u} \leq \tilde{u} \leq \bar{u}$. Therefore $\underline{u}, \bar{u}$ are minimal and maximal $\omega$-periodic mild solutions of Eq. (2.5) and $\underline{u}, \bar{u}$ can be obtained by the iterative sequences defined in (3.4) starting from $\alpha_{0}$ and $\beta_{0}$. This completes the proof of Theorem 3.1.

Now we discuss the uniqueness of time $\omega$-periodic solution of the problem (1.4).

THeOREM 3.2. Let $F: \mathbf{R} \times E \times E \rightarrow E$ is continuous function which is $\omega$-periodic in $t, S: C_{\omega}(\mathbf{R}, E) \rightarrow C_{\omega}(\mathbf{R}, E)$ is a positive linear bounded operator. Suppose that Eq. (2.5) has lower and upper w-periodic solutions $\alpha_{0}, \beta_{0} \in C_{\omega}^{1}(\mathbf{R}, E) \cap$ $C_{\omega}\left(\mathbf{R}, E_{1}\right)$ with $\alpha_{0} \leq \beta_{0}$. Assume that conditions $(A 1)$, (A2) and following conditions

(A3) there are positive constants $L_{1}, L_{2}$ such that

$$
F\left(t, w_{2}, v_{2}\right)-F\left(t, w_{1}, v_{1}\right) \leq L_{1}\left(w_{2}-w_{1}\right)+L_{2}\left(v_{2}-v_{1}\right)
$$

for all $t \in \mathbf{R}, \alpha_{0}(t) \leq w_{1} \leq w_{2} \leq \beta_{0}(t), S \alpha_{0}(t) \leq v_{1} \leq v_{2} \leq S \beta_{0}(t)$,

(A4) there is positive constant $L_{3}$ such that

$$
S w_{2}-S w_{1} \leq L_{3}\left(w_{2}-w_{1}\right),
$$

for all $w_{1}, w_{2} \in\left[\alpha_{0}, \beta_{0}\right]$ and $w_{2} \geq w_{1}$,

hold, then Eq. (2.5) has a unique $\omega$-periodic mild solution $u^{*}$ between $\alpha_{0}$ and $\beta_{0}$ provided that $L_{1}+L_{2} L_{3}<\lambda_{1}\left(\lambda_{1}\right.$ is the first eigenvalue of $\left.A\right)$.

Proof. By Theorem 3.1, we know that the abstract evolution equation (2.5) has minimal and maximal $\omega$-periodic mild solutions $\underline{u}, \bar{u} \in C_{\omega}(\mathbf{R}, E)$, which can 
be obtained by the iterative sequences defined in (3.4) starting from $\alpha_{0}$ and $\beta_{0}$. Therefore, we only show that $\underline{u}(t) \equiv \bar{u}(t)$ for $t \in \mathbf{R}$.

To this end, we arbitrarily choose $u_{1}, u_{2} \in[\alpha, \beta]$ with $u_{1} \leq u_{2}$, thus $u_{1}(t) \leq$ $u_{2}(t)$ and $S u_{1}(t) \leq S u_{2}(t)$ for $t \in \mathbf{R}$, by assumptions $(A 3)$ and $(A 4)$, we can obtain

$$
\begin{aligned}
F(t, & \left.u_{2}(t), S u_{2}(t)\right)-F\left(t, u_{1}(t), S u_{1}(t)\right) \\
& \left.\leq L_{1}\left(u_{2}(t)-u_{1}(t)\right)+L_{2}\left(S u_{2}(t)\right)-S u_{1}(t)\right) \\
& \leq L_{1}\left(u_{2}(t)-u_{1}(t)\right)+L_{2} L_{3}\left(u_{2}(t)-u_{1}(t)\right) \\
& =\left(L_{1}+L_{2} L_{3}\right)\left(u_{2}(t)-u_{1}(t)\right) .
\end{aligned}
$$

Hence, for $\underline{u}, \bar{u} \in\left[\alpha_{0}, \beta_{0}\right]$, which implies that $\underline{u}(t), \bar{u}(t) \in\left[\alpha_{0}(t), \beta_{0}(t)\right]_{E}$, we have

$$
F(t, \bar{u}(t), G \bar{u}(t))-F(t, \underline{u}(t), G \underline{u}(t)) \leq\left(L_{1}+L_{2} L_{3}\right)(\bar{u}(t)-\underline{u}(t)), \quad t \in \mathbf{R} .
$$

Since $\bar{u}, \underline{u}$ are the fixed points of the operator $Q$, then by (3.1) and (3.15), we obtain

$$
\begin{aligned}
\theta & \leq \bar{u}(t)-\underline{u}(t)=(Q \bar{u})(t)-(Q \underline{u})(t) \\
& =P(F(t, \bar{u}(t), S \bar{u}(t))-F(t, \underline{u}(t), S \underline{u}(t))) \\
& \leq\left(L_{1}+L_{2} L_{3}\right) P(\bar{u}(t)-\underline{u}(t)) \leq \cdots \leq\left(L_{1}+L_{2} L_{3}\right)^{n} P^{n}(\bar{u}(t)-\underline{u}(t)) .
\end{aligned}
$$

By the normality of the cone $K_{C}$, we can see

$$
\|\bar{u}-\underline{u}\|_{C} \leq\left(L_{1}+L_{2} L_{3}\right)^{n}\left\|P^{n}\right\| \cdot\|\bar{u}-\underline{u}\|_{C} .
$$

From the spectral radius of Gelfand formula $\lim _{n \rightarrow \infty} \sqrt[n]{\left\|P^{n}\right\|}=r(P)=\frac{1}{\lambda_{1}}$, and the condition $L_{1}+L_{2} L_{3}<\lambda_{1}$, we get that $\left(L_{1}+L_{2} L_{3}\right)^{n}\left\|P^{n}\right\|<1$ when $n$ is large enough, which implies that $\|\bar{u}-\underline{u}\|_{C}=0$, it follows that $\bar{u} \equiv \underline{u}$. Thus, the abstract evolution equation (2.5) has only one $\omega$-periodic mild solution $u^{*}=$ $\bar{u}=\underline{u}$, which can be obtained by a monotone iterative procedure starting from $\alpha_{0}$ or $\beta_{0}$. This completes the proof of Theorem 3.2.

\section{Main results}

In this section, we apply our abstract results to show the existence and uniqueness of periodic solutions for nonlocal delayed parabolic equation (1.4). To end this, we need the following definitions. satisfies

Definition 4.1. If a function $\alpha \in C^{2,1}(\Omega \times \mathbf{R})$, which is $\omega$-periodic in $t$,

$$
\left\{\begin{array}{l}
\frac{\partial \alpha}{\partial t}+A(x, D) \alpha \leq f(x, t, \alpha(x, t), g * \alpha(x, t)), \quad x \in \Omega, t \in \mathbf{R} \\
B \alpha=0, \quad x \in \Omega
\end{array}\right.
$$


we call it an $\omega$-periodic lower solution of Eq. (1.4). If the inequality of (4.1) is inverse, we call it an $\omega$-periodic upper solution of Eq. (1.4).

Now, we are in the position to state and prove our main results.

THEOREM 4.1. Let $f: \Omega \times \mathbf{R} \times \mathbf{R}^{2} \rightarrow \mathbf{R}$ is local Hölder-continuous function which is $\omega$-periodic in $t$, the convolution $g * u(x, t)$ is defined by (1.5). Suppose that Eq. (1.4) has lower and upper w-periodic solutions $\alpha_{0}, \beta_{0} \in C^{2,1}(\Omega \times \mathbf{R})$ with $\alpha_{0} \leq \beta_{0}$. If the following assumptions

(B1) there are two constants $C_{1} \geq 0$ and $C_{2}>0$ such that

$$
f\left(x, t, w_{2}, v_{2}\right)-f\left(x, t, w_{1}, v_{1}\right) \geq C_{1}\left(w_{2}-w_{1}\right)+C_{2}\left(v_{2}-v_{1}\right),
$$

for all $t \in \mathbf{R}, \alpha_{0}(x, t) \leq w_{1} \leq w_{2} \leq \beta_{0}(x, t)$, and $g * \alpha_{0}(x, t) \leq v_{1} \leq v_{2} \leq g * \beta_{0}(x, t)$,

(B2) there is a constant $0 \leq C_{3} \leq \frac{C_{1}}{C_{2}}$ such that

$$
u_{2}(x, t+s)-u_{1}(x, t+s) \geq-C_{3}\left(u_{2}(x, t)-u_{1}(x, t)\right),
$$

for all $s \in(-\infty, 0], t \in \mathbf{R}, u_{1}, u_{2} \in\left[\alpha_{0}, \beta_{0}\right]$ with $u_{2} \geq u_{1}$,

hold, then Eq. (1.4) has minimal and maximal $\omega$-periodic solution $\underline{u}, \bar{u} \in C^{2,1}(\overline{\mathbf{\Omega}} \times \mathbf{R})$ between $\alpha_{0}$ and $\beta_{0}$, which can be obtained by monotone iterative sequences starting from $\alpha_{0}$ and $\beta_{0}$.

Proof. From Section 2, we know that the $\omega$-periodic problem of equation (1.4) can be reformulated as the $\omega$-periodic problem of abstract evolution equation (2.5). Thus, the lower and upper $\omega$-periodic solutions of Eq. (1.4) are the lower and upper $\omega$-periodic solutions of abstract evolution equation (2.5). By the conditions $(B 1),(B 2)$ and (2.4), we can easily prove that the conditions $(A 1)$ and $(A 2)$ hold. Therefore, from Theorem 3.1, we know that abstract evolution equation (2.5) has minimal and maximal $\omega$-periodic mild solutions $\underline{u}, \bar{u}$ and $\underline{u}, \bar{u}$ can be obtained by the iterative sequences defined in (3.4) starting from $\alpha_{0}$ and $\beta_{0}$. By the analyticity of semigroup $T(t)(t \geq 0)$ and the regularization method used in [2], we can see $\underline{u}, \bar{u} \in C^{2,1}(\bar{\Omega} \times \mathbf{R})$ are classical time $\omega$-periodic solutions of the problem (1.4). This completes the proof of Theorem.

THEOREM 4.2. Let $f: \Omega \times \mathbf{R} \times \mathbf{R}^{2} \rightarrow \mathbf{R}$ is local Hölder-continuous function which is w-periodic in $t$, the convolution $g * u(x, t)$ is defined by (1.5). Suppose that Eq. (1.4) has lower and upper $\omega$-periodic solutions $\alpha_{0}, \beta_{0} \in C^{2,1}(\Omega \times \mathbf{R})$ with $\alpha_{0} \leq \beta_{0}$. Assume that conditions (B1), (B2) and following conditions

(B3) there are positive constants $L_{1}, L_{2}$ such that

$$
f\left(x, t, w_{2}, v_{2}\right)-f\left(x, t, w_{1}, v_{1}\right) \leq L_{1}\left(w_{2}-w_{1}\right)+L_{2}\left(v_{2}-v_{1}\right),
$$

for all $t \in \mathbf{R}, \alpha_{0}(x, t) \leq w_{1} \leq w_{2} \leq \beta_{0}(x, t)$, and $g * \alpha_{0}(x, t) \leq v_{1} \leq v_{2} \leq g * \beta_{0}(x, t)$,

(B4) there is positive constant $L_{3}$ such that

$$
u_{2}(x, t+s)-u_{1}(x, t+s) \leq L_{3}\left(u_{2}(x, t)-u_{1}(x, t)\right),
$$


for all $s \in(-\infty, 0], t \in \mathbf{R}, u_{1}, u_{2} \in\left[\alpha_{0}, \beta_{0}\right]$ with $u_{2} \geq u_{1}$,

hold, then Eq. (1.4) has a unique $\omega$-periodic solution $u^{*} \in C^{2,1}(\bar{\Omega} \times \mathbf{R})$ between $\alpha_{0}$ and $\beta_{0}$ provided that $L_{1}+L_{2} L_{3}<\lambda_{1}$ ( $\lambda_{1}$ is the first eigenvalue of $A(x, D)$ under the boundary condition $\mathrm{Bu}=0$ ).

Proof. By Theorem 4.1, we know that we know that the $\omega$-periodic problem of equation (1.4) has minimal and maximal $\omega$-periodic solutions $\underline{u}, \bar{u} \in C^{2,1}(\bar{\Omega} \times \mathbf{R})$, which can be obtained by the iterative sequences defined in (3.4) starting from $\alpha_{0}$ and $\beta_{0}$. On the other hand, we also can obtain that the conditions $(A 3)$ and $(A 4)$ hold from $(B 3)$ and $(B 4)$. Hence, by Theorem 3.2, we can get that Eq. (1.5) has only one $\omega$-periodic mild solution $u^{*}=\bar{u}=\underline{u}$, which implies that $u^{*} \in C^{2,1}(\bar{\Omega} \times \mathbf{R})$ is a unique classical time $\omega$-periodic solution of the problem (1.4). This completes the proof of Theorem.

\section{REFERENCES}

[1] S. Ahmad AND A. C. Lazer, Asymptotic behaviour of solutions of periodic competition diffusion systems, Nonlinear Anal. 13 (1989), 263-284.

[2] H. Amann, Periodic solutions of semilinear parabolic equations, Nonlinear analysis (L. Cesari, R. Kannan and R. Weinberger, eds.), A collection of papers in honor of Erich Rothe, Academic Press, New York, 1978, 1-29.

[ 3 ] N. Britton, Aggregation and the competitive exclusion principle, J. Theor. Biol. 136 (1989), 57-66.

[4] N. BRITTON, Spatial structures and periodic travelling waves in an integro-differential reactiondiffusion population model, SIAM J. Appl. Math. 50 (1990), 1663-1688.

[5] T. A. Burton ANd B. Zhang, Periodic solutions of abstract differential equations with infinite delay, J. Differ. Equ. 90 (1991), 357-396.

[6] C. D. Coster AND P. OMARI, Unstable periodic solutions of a parabolic problem in the presence of non-well-order lower and upper solutions, J. Funct. Anal. 175 (2000), 5288.

[7] S. A. Gourely and J. W. H. So, Dynamics of a food-limited population model incorporating nonlocal delays on a finite domain, J. Math. Biol. 44 (2002), 49-78.

[8] M. X. HE, On periodic and almost periodic solutions of reaction diffusion system with time lag, Acta Math. Sinica (Chin. Ser.) 32 (1989), 91-97.

[9] D. HenRy, Geometric theory of semilinear parabolic equations, Lecture notes in math. 840, Springer-Verlag, New York, 1981.

[10] Y. LI, The positive solutions of abstract semilinear evolution equations and their applications, Acta Math. Sin. 39 (1996), 666-672 (in Chinese).

[11] Y. LI, The global solutions of initial value problems for abstract semilinear evolution equations, Acta Anal. Funct. Appl. 3 (2001), 339-347 (in Chinese).

[12] Y. LI, Existence and uniquness of positive periodic solution for abstract semi-linear evolution equations, J. Sys. Sci. Math. Scis. 25 (2005), 720-728 (in Chinese).

[13] Y. LI, Existence and asymptotic stability of periodic solution for evolution equations with delays, J. Funct. Anal. 261 (2011), 1309-1324.

[14] G. M. Lieberman, Time-periodic solutions of quasilinear parabolic equations, J. Math. Anal. Appl. 264 (2001), 617-638.

[15] G. LIN AND W. T. LI, Bistable wavefronts in a diffusive and competitive Lotka-Volterra type system with nonlocal delays, J. Differ. Equ. 244 (2008), 487-513. 
[16] X. Lu AND W. Feng, Periodic solution and oscillation in a competition model with diffusion and distributed delay effect, Nonlinear Anal. 27 (1996), 699-709.

[17] S. MA, Traveling waves for non-local delayed diffusion equations via auxiliary equation, J. Differ. Equ. 237 (2007), 259-277.

[18] C. OU AND J. Wu, Persistence of wavefronts in delayed nonlocal reaction-diffusion equations, J. Differ. Equ. 235 (2007), 219-261.

[19] C. V. PAO, Periodic solutions of parabolic systems with nonlinear boundary conditions, J. Math. Anal. Appl. 234 (1999), 695-716.

[20] C. V. PaO, Periodic solutions of parabolic systems with time delays, J. Math. Anal. Appl. 251 (2000), 251-263.

[21] A. PAZY, Semigroup of linear operators and applications to partial differential equations, Applied mathematical sciences 44, Springer, New York, NY, USA, 1983.

[22] A. Tineo and J. Rivero, Permanence and asymptotic stability for competitive and LotkaVolterra systems with diffusion, Nonlinear Anal. Real World Appl. 4 (2003), 615-624.

[23] C. WANG, Existence and stability of periodic solutions for parabolic systems with time delays, J. Math. Anal. Appl. 339 (2008), 1354-1361.

[24] Z. C. WANG, W. T. Li AND S. RuAN, Travelling wave fronts in reaction-diffusion systems with spatio-temporal delays, J. Differ. Equ. 222 (2006), 185-232.

[25] L. WANG AND Z. WANG, Monotone iterative technique for parameterized BVPs of abstract semilinear evolution equations, Comput. Math. Appl. 46 (2003), 1229-1243.

[26] Y. YAmADA AND Y. NIIKURA, Bifurcation of periodic solutions for nonlinear parabolic equations with infinite delay, Funkcial Ekvac. 29 (1986), 309-333.

[27] Y. Yang, R. Zhang, J. YIN AND C. JIN, Existence of time periodic solutions for the Nicholsons blowflies model with Newtonian diffusion, Math. Methods Appl. Sci. 33 (2010), 922-934.

[28] J. M. ZhU, Y. C. LiU And Z. X. LI, The existence and attractivity of time periodic solutions for evolution equations with delays, Nonlinear Anal. Real World Appl. 9 (2008), 842-851.

Qiang Li

Department of Mathematics

NORTHWEST NORMAL UNIVERSITY

LANZHOU 730070

P.R. CHINA

E-mail: lznwnuliqiang@126.com

Yongxiang $\mathrm{Li}$

Department of Mathematics

NoRTHWEST NORMAL UNIVERSITY

LANZHOU 730070

P.R. ChINA

E-mail: liyx@nwnu.edu.cn

Pengyu Chen

Department of Mathematics

NORTHWEST NORMAL UNIVERSITY

LANZHOU 730070

P.R. CHINA

E-mail: chpengyu123@163.com 